\title{
Piper nigrum Fruit Extract Prevents TMA-Induced Allergic Contact Dermatitis by Regulating Th2 Cytokine Production
}

\author{
Sung Keun Jung ${ }^{1,3,+}$, Dae Woon Choi ${ }^{1,+}$, Chang Hwa Jung ${ }^{2,3}$, Yang-Ji Kim ${ }^{2,3}$, Sun Young Jung ${ }^{1}$ \\ \& Dong-Hwa Shon ${ }^{1,3}$ \\ ${ }^{1}$ Research Group of Food Functionality, Korea Food Research Institute, Seongnam, Republic of Korea \\ ${ }^{2}$ Research Group of Nutrition and Metabolic System, Korea Food Research Institute, Seongnam, Republic of \\ Korea \\ ${ }^{3}$ Food Biotechnology Program, Korea University of Science and Technology, Daejeon, Republic of Korea \\ Correspondence: Dong-Hwa Shon, Research Group of Food Functionality, Division of Platform Technology \\ Research, Korea Food Research Institute, 1201-62, Seongnam, Republic of Korea. Tel: 82-031-780-9133. \\ E-mail: dhs95@kfri.re.kr
}

${ }^{\dagger}$ These authors are equally contributed to this work.

Received: November 9, 2014 Accepted: December 4, 2014 Online Published: January 15, 2015

doi:10.5539/jas.v7n2p135 URL: http://dx.doi.org/10.5539/jas.v7n2p135

\begin{abstract}
Allergic contact dermatitis (ACD) remains a leading skin disease in many countries. In this study, we investigated the preventive effect of Piper nigrum fruit extract (PFE) on trimellitic anhydride (TMA)-induced dermatitis and its potential mechanism of action. Oral administration of PFE and prednisolone (PS) significantly suppressed TMA-induced increases in ear, epidermal thickness, and infiltration of $\mathrm{CD} 4^{+}$cells, while abnormal expression of IgE, mMCP-1, IL-1 $\beta$ and IL- $1 \beta$ mRNA was also significantly counteracted by oral administration of PFE. PFE also significantly suppresses TMA-induced IL-4 and IL-5 production and IL-4 mRNA expression in vivo, as well as OVA-induced IL-4, IL-5, and IL-13 production and GATA3 mRNA expression ex vivo, and IL-4 induced STAT6 phosphorylation in primary cultured splenocytes and HaCaT cells. Interestingly, the PFE component piperine significantly suppressed OVA-induced IL-4, IL-5, and IL-13 secretion ex vivo. Taken together, these results suggest that PFE could be useful in suppressing allergic contact dermatitis.
\end{abstract}

Keywords: Piper nigrum fruit, allergic contact dermatitis, Th2 cytokines, STAT6, piperine

\section{Introduction}

Allergic contact dermatitis (ACD) arises from a delayed type IV hypersensitivity response and is one of the most common skin diseases, affecting $15-20 \%$ of the general population worldwide (Peiser et al., 2012). Compounds lower than 500 daltons have been observed to penetrate through the stratum corneum barrier of epidermal skin, where they become haptens (Martin, 2004). After haptenization, the generation of immunogenic neo-antigens occurs via binding between self-proteins and hapten compounds, as well as hapten and hapten-protein complexes recognized by the innate immune system as 'altered self', leading to the induction of several pro-inflammatory mediators, including interleukin-1 $\beta$ (IL-1 $\beta$ ) (Kaplan et al., 2012). Following these events, haptenated skin-resident dendritic cells (DCs) or DCs with acquired haptenated proteins migrate to skin-draining lymph nodes, and peptides from haptenated cytokines act as signaling transducers which activate the signal transducer and activator of transcription (STAT), subsequently regulating T cell differentiation.

$\mathrm{T}$ helper (Th) lymphocytes differentiate into Th1, Th2, Th17, and regulatory $\mathrm{T}$ (Treg) cells, and play an important role in the serial adaptive immune response to various infectious agents through the production of specific cytokines (Szabo et al., 2000; Mosmann et al., 2005). Th1 cells secrete interferon gamma (IFN- $\gamma$ ) and protect their host against intracellular pathogens and viruses (Szabo et al., 2000). Th2 cells produce interleukin 4 (IL-4), IL-5 and IL-13, and support the role of B cells in removing parasites (Szabo et al., 2000). Th17 cells act to protect the body against bacterial and fungal infection by producing IL-17, IL-21, and IL-22 (Furuzawa-Carballeda et al., 2007). Additionally, Treg cells play a critical role in the regulation of immune cell homeostasis by producing IL-10 and transforming growth factor-beta (TGF- $\beta$ ) (Kim et al., 2013). 
The differentiation of $\mathrm{CD}^{+} \mathrm{T}$ cells and their subsequent function in regulating immune responses is tightly regulated by specific cytokine profiles and transcriptional signaling (Murphy \& Stockinger, 2010). IL-12 and IFN- $\gamma$ are the two primary cytokines involved in Th1 differentiation. For Th2 differentiation, IL-4, IL-5, and IL-13 are involved. In turn, the phosphorylation of STAT plays a central role in the regulation of cytokine production ( $\mathrm{Zhu}$ et al., 2010). Once IL-4 binds to its cognate receptor (the IL-4 receptor), STAT6 phosphorylation occurs and subsequently activates lineage-specific master transcription factors, particularly the GATA3 binding protein 3 (GATA-3).

The consumption of natural foods has been linked to the prevention or alleviation of abnormal immune responses to foreign pathogens and products, including hapten compounds (Jin et al., 2011; E. Cho \& S. H. Cho, 2013; Choi et al., 2014). Piper nigrum extracts have been used as both dietary ingredient and traditional herbal medicine and shown to exhibit various biological properties, including antibacterial activity (Paulkumar et al., 2014) and counteracting memory impairment (Hritcu et al., 2014). Additionally, HPLC-MS approaches have identified several compounds from Piper nigrum including pellitorine, piperylin, 4,5-dihydropiperlonguminine, piperlonguminine, 4,5-dihydropiperine, piperine, and pipercide (Scott et al., 2005). A previous study has shown that oral administration of a methanolic extract of Piper nigrum leaf suppresses dinitrofluorobenzene (DNFB)-induced immune reactions in vivo and histamine release by rat peritoneal mast cells (Hirata et al., 2008). However, the effect of Piper nigrum fruit extract (PFE) on hapten-induced dermatitis and its mechanism of action have not been explored in detail.

In the present study, we investigated the preventive effect of Piper nigrum fruit extract on TMA-induced dermatitis using a murine ACD model. We observed that PFE inhibited TMA-induced ear swelling, increases in epidermal thickness and $\mathrm{CD}^{+}$cell infiltration. Furthermore, PFE exerted inhibitory effects on IgE, mMCP-1, and IL- $1 \beta$ production, as well as IL-1 $\beta$ mRNA expression and Th2 cytokine production. IL-4, IL-5, and IL-13 expression induced by TMA or OVA arose due to the suppression of STAT6 phosphorylation and GATA3 mRNA expression. These findings suggest that PFE could be potentially used as a strong preventive-ACD agent that inhibits Th2 cytokine production via the suppression of STAT6/GATA3 signaling.

\section{Materials and Methods}

\subsection{Materials}

TMA, prednisolone, ovalbumin (OVA, Grade VI), piperine, and piperonal were purchased from Sigma-Aldrich (St. Louis, Mo, USA). Inject Alum was obtained from Pierce Biotechnology (Rockford, IL, USA). Recombinant mouse IL-4 and human IL-4 were purchased from BD Biosciences (San Diego, CA, USA). Antibodies specific for STAT6, CD4, $\beta$-actin, horseradish peroxidase (HRP)-conjugated goat anti-rabbit, anti-mouse and DyLight 594-conjugated goat anti-rabbit IgG were purchased from Santa Cruz (CA, USA). Antibodies for mouse phospho-STAT6 were obtained from Abcam (Cambridge, UK) and human phospho-STAT6 was purchased from Cell Signaling (Danvers, MA, USA).

\subsection{Sample Preparation}

Black pepper was purchased from a rural market (Gyeonggi-do, Korea). The extract was prepared using a soxhlet flux extractor. Briefly, the black pepper was incubated in $70 \%$ ethanol at $65{ }^{\circ} \mathrm{C}$ for two periods of $3 \mathrm{hrs}$ each. The $70 \%$ ethanol extract was filtered using filter paper (Whatman no. 4), before the extract was concentrated by rotary evaporator (IKA RV10, USA) and dried in a freeze dryer. The dried black pepper extract (PFE) was stored at $4{ }^{\circ} \mathrm{C}$.

\subsection{Animals}

Animals were maintained under specific pathogen-free conditions following established guidelines and the experimental protocol (KFRI-M-14013) was approved by the Animal Care and Use Committee of Korea Food Research Institute. 5-week-old female BALB/c mice were purchased from OrientBio Inc. (Gyeonggi-do, Korea) and were housed in an air-conditioned room $\left(23 \pm 2{ }^{\circ} \mathrm{C}\right)$ with a $12 \mathrm{~h} \mathrm{light} /$ dark cycle and treated in accordance with the Korea Food Research Institute guidelines for animal care and use.

\subsection{Induction of Contact Hypersensitivity and Experimental Schedule}

Induction of CHS was achieved by modifying a chronic CHS model (Schneider et al., 2009). Briefly, 6-week-old mice were divided into four groups: naive group (naive), sham group (sham), PFE $50 \mathrm{mg} / \mathrm{kg}$ group (PFE 50), PFE $200 \mathrm{mg} / \mathrm{kg}$ group (PFE 200), and a $10 \mathrm{mg} / \mathrm{kg}$ prednisolone group (PS 10). PFE and prednisolone were orally administrated daily from Day -7 . After 1 week, mice were sensitized with $50 \mu \mathrm{L}$ of $5 \%$ TMA in mixed solvent (acetone/isopropyl myristate, 4:1. v/v) on their shaved right flank on Day 0 and $10 \mu \mathrm{L}$ of $5 \%$ TMA was administered daily on the dorsum of both ears from Day 5 to 14 . Ear thickness was measured $24 \mathrm{~h}$ after TMA 
treatment on Day 5-14 using a custom-built micrometer (Schering AG, Germany).

\subsection{Sensitization and Challenge with OVA and Preparation of Splenocyte Cultures}

$\mathrm{BALB} / \mathrm{c}$ mice (female, 6 weeks old) were sensitized by intraperitoneal (i.p.) injection with $10 \mu \mathrm{g}$ ovalbumin adsorbed in $1 \mathrm{mg}$ of Inject Alum on Days 0 and 7. After 1 week, splenocytes were prepared by aseptically removing the spleens from the mice. Homogenized single spleen cells were collected and treated with red blood cell (RBC)-lysing buffer (Sigma-Aldrich, St. Louis, MO, USA). Splenocytes were diluted to $5 \times 10^{6} \mathrm{cells} / \mathrm{mL}$ in RPMI 1640 media containing $10 \% \mathrm{FBS}, 100 \mathrm{U} / \mathrm{ml}$ penicillin and $100 \mathrm{mg} / \mathrm{ml}$ streptomycin. The splenocytes were then cultured in the presence or absence of OVA $(100 \mu \mathrm{g} / \mathrm{mL})$ with samples. The plates were incubated at $37{ }^{\circ} \mathrm{C}$ for $48 \mathrm{~h}$ (for real-time PCR analysis) or $72 \mathrm{~h}$ (for ELISA analysis) in a humidified incubator with $5 \%(\mathrm{v} / \mathrm{v}) \mathrm{CO}_{2}$ and $95 \%(\mathrm{v} / \mathrm{v})$ air.

\subsection{Cytokine Measurement by ELISA Assay}

For cytokine measurement in the ear tissue of BALB/c mice, ears were mechanically homogenized in $2 \mathrm{~mL}$ of lysis buffer (Cell Signaling, Danvers, MA, USA) containing a protease inhibitor cocktail (Roche, Indianapolis, IN), and centrifuged at $25,000 \mathrm{~g}$ for $30 \mathrm{~min}$ at $4{ }^{\circ} \mathrm{C}$. The concentration of total protein in the supernatant was calculated by protein assay kit (Bio-Rad Laboratories, USA).

Cytokine levels in ear supernatant and splenocyte culture medium were measured using IL-4, IL-5, IL-1 $\beta$ (BD Biosciences, San Diego, CA, USA), and IL-13 (R\&D systems, Minneapolis, MN, USA) ELISA kits. Total-IgE and mMCP-1 in serum was also detected with an IgE (BD Bioscience, San Diego, CA, USA) and an mMCP-1 ELISA kit (eBioscience, San Diego, CA). All ELISAs were conducted in accordance with the manufacturer's instructions.

\subsection{RNA Isolation and Quantitative Real-Time PCR Analyses}

Total RNA in tissues and splenocytes was recovered and purified using an RNeasy ${ }^{\circledR}$ Mini Kit (Qiagen, Germany) according to the manufacturer's instructions. cDNA was synthesized using a QuantiTect Reverse Transcription Kit (Qiagen, Germany). First-strand cDNA was prepared from $1 \mu \mathrm{g}$ of total RNA. The samples were subjected to real-time PCR using SYBR ${ }^{\circledR}$ Green Master Mix in a Rotor-Gene Q 2plex System (Qiagen, Germany). The gene expression levels were normalized by comparing with GAPDH expression. Relative gene expression changes, calculated using the 2-delta CT method, are reported as fold change compared to that of the control samples. Primers used in this experiment were purchase from Bioneer (Daejeon, Korea).

\subsection{Western Blot Analysis}

To analyze the effects of PFE on IL-4-induced STAT6 phosphorylation, splenocytes were prepared by aseptically removing the spleens of BALB/c mice (female, 6 weeks old) and incubating with $2 \mu \mathrm{g}$ concanvalin A (Con A, Sigma-Aldrich, St. Louis, MO, USA) for 2 days. Splenocytes were centrifuged at 1,300 rpm for 5 min and supernatant was removed, before incubation in RPMI 1640 media in the absence of Con A. After 1 day, the splenocytes were pre-incubated with PFE for $1 \mathrm{~h}$. before stimulation with IL-4 (BD Biosciences, San Diego, CA, USA) for 15 min. For human keratinocyte analysis, immortalized human epidermal keratinocyte HaCaT cells were maintained at $37{ }^{\circ} \mathrm{C}$ in Dulbecco's modified Eagle's medium (DMEM) containing $10 \%$ fetal bovine serum, $100 \mathrm{U} / \mathrm{ml}$ penicillin, and $100 \mathrm{mg} / \mathrm{ml}$ streptomycin in a $5 \% \mathrm{CO}_{2}$ humidified incubator. The $\mathrm{HaCaT}$ cells were pre-incubated with PFE for $1 \mathrm{~h}$ prior to stimulation with IL-4 (BD Biosciences, San Diego, CA, USA) for 15 $\min$.

Protein concentration was determined using a dye-binding protein assay kit (Bio-Rad Laboratories, USA) following the instructions in the manufacturer's manual. Lysate protein was subjected to $10 \%$ sodium dodecyl sulfate-polyacrylamide gel electrophoresis (SDS-PAGE) and transferred to a polyvinylidene difluoride (PVDF) membrane (Amersham Pharmacia Biotech). After transferring, the membranes were incubated with specific primary antibodies at $4{ }^{\circ} \mathrm{C}$ overnight. Protein bands were visualized using an AE-9300 Ez-Capture MG (ATTO Corporation, Japan) after hybridization with a horseradish peroxidase (HRP)-conjugated secondary antibody.

\subsection{Measurement of Epidermal Thickness}

Excised ears were embedded in $10 \%$ formaldehyde and cut into $6-\mu \mathrm{m}$-thick sections under a microscope (CryostatCM3050S, Leica Microsystems, Germany). The sections were stained with hematoxylin and 0.5\% eosin (Sigma; hematoxylin and eosin [H\&E] staining) to measure the epidermal thickness of the ear tissue samples. Thickness was analyzed using Micrometrics SE Premium software.

\subsection{Immunofluorescence Analysis}

Five $\mu$ m-thick sections of paraffin-embedded mice ear tissues were cut using a microtome and air-dried at room 
temperature overnight. Paraffin sections were de-paraffinized using xylene and hydrated in descending grades of ethanol in distilled water, after which antigen retrieval was performed by heating samples at $95-100^{\circ} \mathrm{C}$ in $10 \mathrm{mM}$ citrate buffer at $\mathrm{pH} 6$ for $10 \mathrm{~min}$. For immunofluorescence, sections from mice ear were permeabilized and blocked at room temperature by incubating in PBS containing $0.02 \%$ Tween 20 and $1 \%$ BSA for $1 \mathrm{~h}$. Incubation with a primary antibody diluted in PBS containing 3\% BSA was conducted at $4{ }^{\circ} \mathrm{C}$ overnight. A secondary Texas red (Santa Cruz Biotechnology) antibody was added at a 1:1000 dilution for $1 \mathrm{~h}$ at room temperature. DAPI $(1: 10,000)$ was used as a nuclear stain. Sections were examined and photographed using either a Leica DM5000B fluorescence microscope (Leica Germany) or a Zeiss LSM 510s confocal microscope (Zeiss Germany). Images were processed using Photoshop (Adobe) software.

\subsection{Statistical Analysis}

Where appropriate, data are expressed as the mean \pm S.E.M., and significant differences were determined using one-way ANOVA (Analysis Of Variance). A probability value of $p<0.05$ was used as the minimum threshold for statistical significance.

\section{Results}

3.1 Effects of BPE on TMA-Induced Ear Swelling and Increases in Epidermal Thickness and CD4 $4^{+}$Cell Infiltration in Mouse Ear Tissue

A

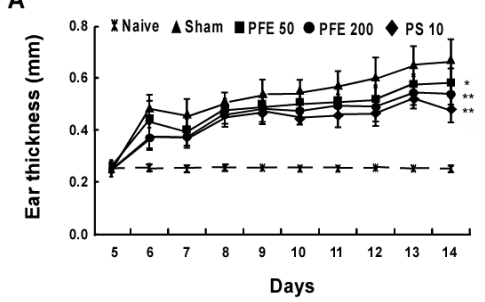

B

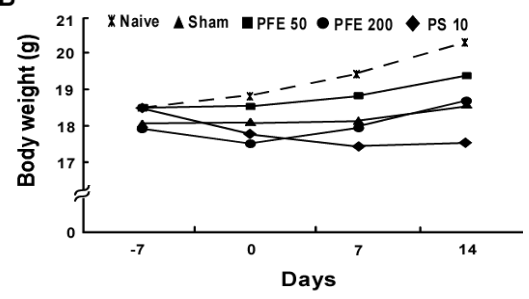

C

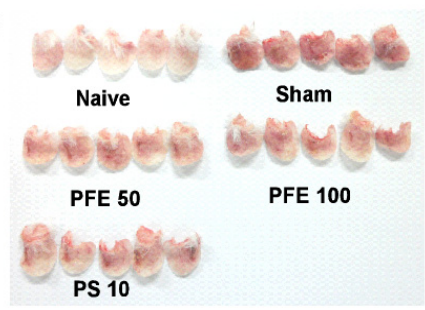

Figure 1. Effect of PFE on TMA-treated ear thickness and weight

(A) PFE significantly suppressed TMA-induced increases in ear thickness in mice. Experimental ACD was induced in the ears of BalB/c mice by TMA treatment for 9 days. After sensitization with TMA, vehicle control, PFE $50 \mathrm{mg} / \mathrm{kg}$, PFE $250 \mathrm{mg} / \mathrm{kg}$, or prednisolone $10 \mathrm{mg} / \mathrm{kg}$ were orally administrated daily for 2 weeks. Ear swelling was evaluated every second day for 9 days. (B) PFE does not affect mouse body weight. The body weights of the mice were measured each week for 3 weeks. (C) Representative image showing the anti-ACD effect of PFE. Images of the mouse ears were recorded using a digital camera (Samsung, Korea) after the mice were euthanized at the end of the experiment. Images shown are representative of those from 10 mice.

To investigate the effects of PFE on allergic contact dermatitis (ACD), we conducted a TMA-induced chronic ACD study (Schneider et al., 2009). Prednisolone (PS) was used as a positive control. Repeated treatment of TMA was observed to cause increases in ear thickness, while the oral administration of PFE and PS significantly suppressed this effect (Figure 1A). As previously reported, PS treatment gradually caused reductions in body weight, but PFE treatment did not (Figure 1B). Observations of mice ear appearance determined that PFE inhibits TMA-induced expansion of blood vessels and redness (Figure 1C). Increases in epidermal thickness and $\mathrm{T}$ cell infiltration are representative symptoms of allergic contact dermatitis. We therefore sought to measure epidermal thickness as well as infiltration of $\mathrm{CD}^{+}$cells, regarded as a marker of $\mathrm{T}$ helper cell activity. H\&E staining results showed that TMA treatment increased epidermal thickness (Figure 2A). Immunofluoresence analysis also showed that chronic application of TMA on mice ears increased the infiltration of $\mathrm{CD} 4^{+}$cells, while oral administration of PFE and PS strongly suppressed these effects in vivo (Figure 2B). 

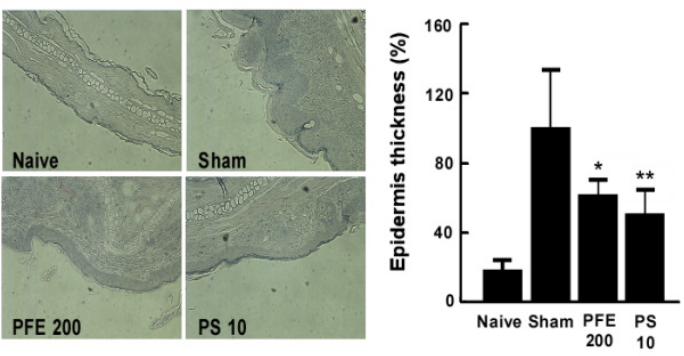

A

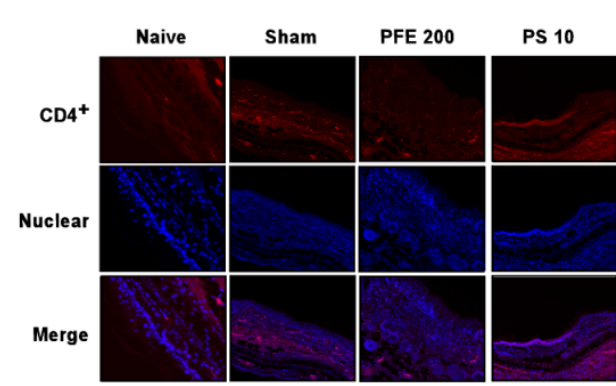

B

Figure 2. Effects of PFE on TMA-treated epidermal thickness and $\mathrm{CD} 4^{+}$cell infiltration in mouse ears

(A) PFE inhibits TMA-induced increases in mouse ear tissue epidermal thickness. Hematoxylin- and eosin-stained images of TMA-treated mouse ears. Images are representative of results from 10 tissue samples. After mice were treated as described for Figure 1A, the ears were excised, sectioned, mounted onto slides, and stained with hematoxylin and eosin for measurement of epidermal thickness. Bars represent the \% relative thickness of epidermis versus the TMA-treated group from 10 animals. Results are shown as mean \pm SEM $(n=$ 5). Asterisks $(*)$ and $(* *)$ indicate significant differences of $p<0.05$ or $p<0.01$, respectively, between the PFE-treated and non-treated groups of TMA-treated mice. (B) PFE inhibits TMA-induced infiltration of CD4 cells into the mouse ear. After mice were treated as described for Figure 1A, the ear tissues were mounted as described for Figure 2A and immunofluorescence assays were conducted to detect $\mathrm{CD}^{+}$positive cells. Representative photos of a minimum of 3 replicates are shown. $\mathrm{CD}^{+}$positive cells are evident as red staining and nuclei were counterstained with DAPI (blue).

\subsection{Effect of PFE on TMA-Induced IgE, mMCP-1, and IL-1 $\beta$ Production, and IL-1 $\mathrm{mRNA}$ Expression Levels in} vivo

Since IgE antibodies are among the first defense molecules to respond to pathogens (Gould \& Sutton, 2008) and increased IgE levels have been detected in ACD patients (Tokura, 2010), we investigated the effect of PFE on TMA-induced IgE levels in mouse serum. The results showed that abnormal increases in IgE levels after treatment of TMA were significantly suppressed by oral administration of PFE, whereas PS treatment had no such effect (Figure 3A). MCP-1 synthesis and secretion has been found to be closely related to IgE expression (Eglite et al., 2003). Thus, we sought to determine whether PFE affects MCP-1 expression. PFE suppressed TMA-induced mMCP-1 expression in mouse serum (Figure 3B). Additionally, PFE administration significantly suppressed IL-1 $\beta$, a pro-inflammatory cytokine, in terms of both mRNA and protein expression (Figures $3 \mathrm{C}$ and 3D). 
A

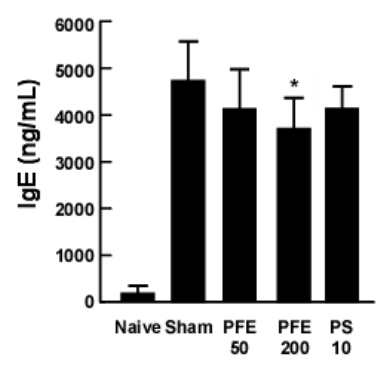

C

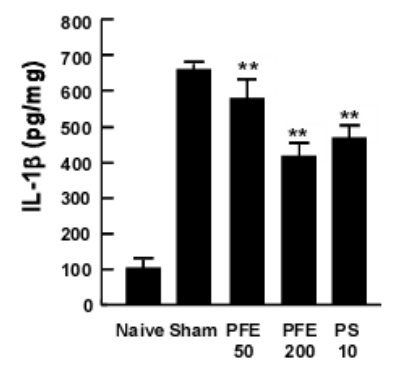

B

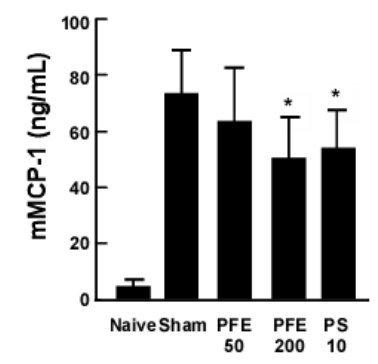

D

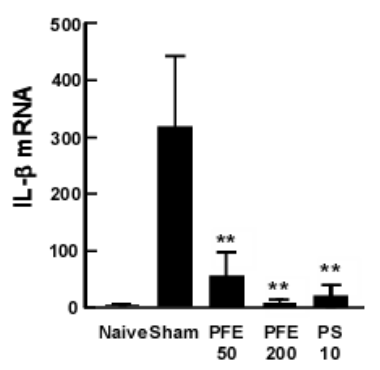

Figure 3. Effect of PFE on TMA-induced IgE, mMCP-1, and IL-1 $\beta$ production and IL-1 $\beta$ mRNA expression in mouse ears

PFE inhibits TMA-induced IgE (A), mMCP-1 (B), and IL-1 $\beta$ (C) expression in mouse ears. After mice were treated as described for Figure 1A, the ears were excised and proteins extracted with buffer for ELISA. Recovered cytokine content was analyzed by ELISA. (D) PFE inhibits TMA-induced IL-1 $\beta$ mRNA expression. After mice were treated as described for Figure 1A, the ears were excised and extraction performed with RNA extraction buffer. IL-1 $\beta$ mRNA expression was detected using RT-PCR as described in the Materials and Methods. Results are shown as mean $\pm \operatorname{SEM}(n=5)$. Asterisks $\left({ }^{*}\right)$ and $(* *)$ indicate significant differences of $p$ $<0.05$ or $p<0.001$, respectively, between the PFE-treated and non-treated groups of TMA-treated mice. Data are representative of three independent experiments that gave similar results.

\subsection{Effects of PFE on TMA-Induced Th2 Cytokine Expression}

Dysregulated Th1 or Th2 cells populations producing aberrant levels of cytokines is one of the major causes of allergic contact dermatitis (Bieber, 2008). Due to the observation that ELISA analysis with ear tissue extract showed that TMA does not affect IFN- $\gamma$ production (data not shown), we excluded the possibility that Th1 cytokines are involved in TMA-induced allergic contact dermatitis. Repetitive TMA treatment of mice ears increased IL-4 and IL-5 levels, which was in turn suppressed by oral administration of PFE (Figures 4A and 4B). IL-4 mRNA expression was also suppressed by oral administration of PFE (Figure 4C). 
A

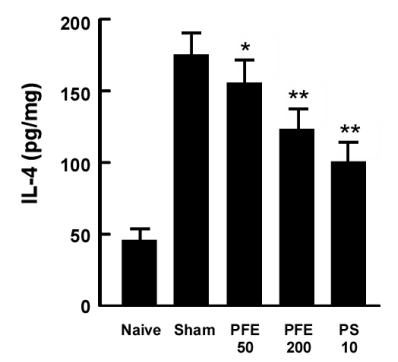

B

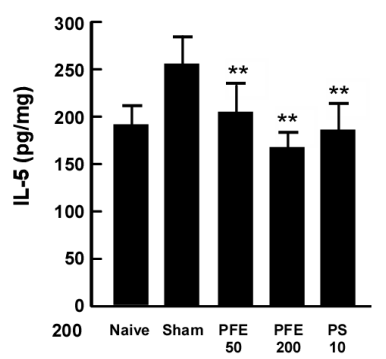

C

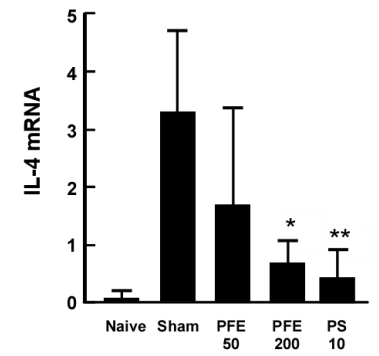

Figure 4. Effect of PFE on TMA-induced IL-4 and IL-5 production and IL-4 mRNA expression in mouse ears

PFE suppresses TMA-induced IL-4 (A) and IL-5 (B) production in mouse ears. After mice were treated as described for Figure 1A, the ears were excised and proteins extracted with buffer for ELISA. Recovered cytokine content was analyzed by ELISA. (B) PFE suppresses TMA-induced IL-4 mRNA expression in mouse ears. After mice were treated as described for Figure 1A, the ears were excised and extracted with RNA extraction buffer. IL-4 mRNA expression was detected by RT-PCR. Data are presented as the mean \pm SD of three independent experiments. Asterisks $(*)$ and $(* *)$ indicate significant differences of $p<0.05$ and $p<0.01$, respectively, between groups with and without PFE.

3.4 Effects of PFE on OVA-Induced Th2 Cytokine and GATA3 mRNA Expression in Primary Cultured Mouse Splenocytes and IL-4 Induced STAT-6 Phosphorylation in Primary Cultured Mouse Splenocytes and Immortalized Human Keratinocytes

The OVA-induced mouse model is well-suited for studying allergenicity with compounds of natural origin (Savinko et al., 2013). We observed that PFE significantly suppresses OVA-induced production of Th2 cytokines, including IL-4, IL-5, and IL-13 (Figures 5A-5C). Additionally, PFE significantly suppressed OVA-induced GATA3 mRNA expression (Figure 5D). STAT plays a critical role in Th cell differentiation via the regulation of GATA3, a Th2 master transcription factor. To investigate the effect of PFE on IL-4-induced STAT6 signaling, we performed Western blot analysis with two cell lines. The results show that PFE strongly suppresses IL-4-induced STAT6 phosphorylation in both splenoyctes and $\mathrm{HaCaT}$ cells (Figures 5E and 5F).

\subsection{Effects of PFE Compounds on OVA-Induced Th2 Cytokine Production in Primary Cultured Mouse Splenocytes}

We sought to determine which component of PFE exerts the major inhibitory effect on OVA-induced Th2 cytokine production in primary cultured mouse splenocytes. The results revealed that piperine significantly suppresses OVA-induced IL-4, IL-5, and IL-13 production, but piperonal does not (Figure 6). 
A

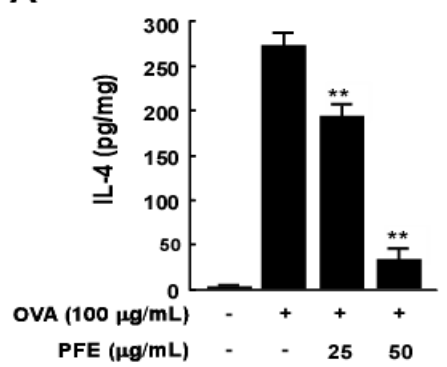

D

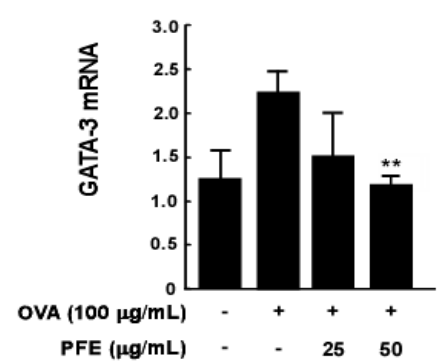

B

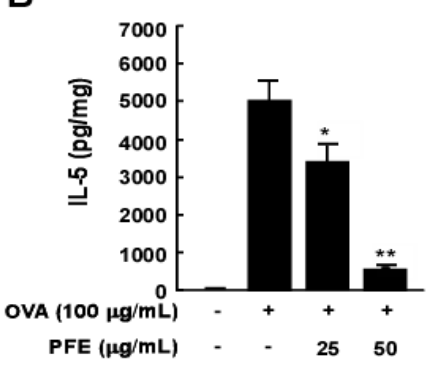

E

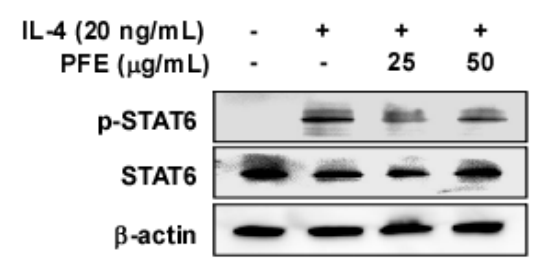

C

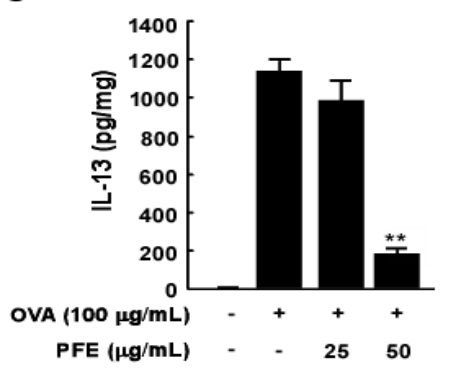

$\mathbf{F}$

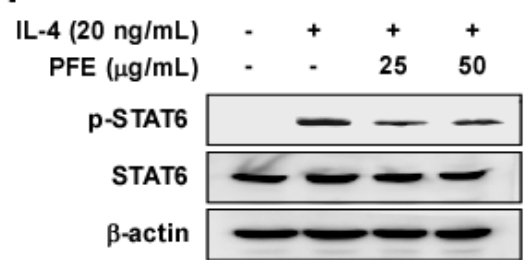

Figure 5. Effects of PFE on OVA-induced Th2 cytokine production and GATA3 mRNA expression in primary cultured splenocytes and IL-4 induced STAT6 phosphorylation in primary cultured splenocytes and human keratinocytes

PFE suppresses OVA-induced IL-4 (A), IL-5 (B) and IL-13 (C) production. After sensitization of mice with OVA, homogenized spleen cells were incubated with PFE and PS in the presence or absence of OVA, and culture media was analyzed by ELISA to detect levels of IL-4, IL-5, and IL-13 cytokines. (D) PFE suppresses OVA-induced GATA3 mRNA expression in primary cultured splenocytes. After mice were treated as described for Figure 5A, protein from cultured homogenized single cells was extracted using RNA extraction buffer. GATA3 mRNA expression was detected by RT-PCR. Data are presented as the mean \pm SD of three independent experiments. Asterisks $\left({ }^{*}\right)$ and $(* *)$ indicate significant differences of $p<0.05$ and $p<0.01$, respectively, between groups with and without PFE. (E) PFE inhibits IL-4-induced STAT6 phosphorylation in primary cultured splenocytes. After mice were treated as described for Figure 5A, homogenized spleen single cells were cultured with concanvalin A for 2 days. PFE was pretreated to the cultured splenocytes, $1 \mathrm{~h}$ before treatment of IL-4 for $15 \mathrm{~min}$, at the indicated concentrations ( $25 \mathrm{and} 50 \mu \mathrm{g} / \mathrm{mL}$ ) for $1 \mathrm{~h}$, again stimulated with IL-4 treatment, and harvested 15 min later. (F) PFE inhibits IL-4-induced STAT6 phosphorylation in human keratinocytes. Cells were pretreated with PFE at the indicated concentrations $(25$ and $50 \mu \mathrm{g} / \mathrm{mL})$ for $1 \mathrm{~h}$, stimulated with IL-4 treatment, and harvested 15 min later. Phosphorylation and expression of STAT6 levels were determined by Western blotting with specific antibodies. Data are representative of three independent experiments that gave similar results. 
A

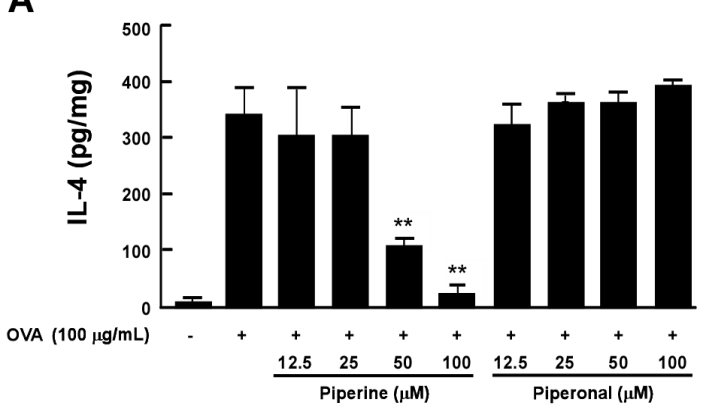

B

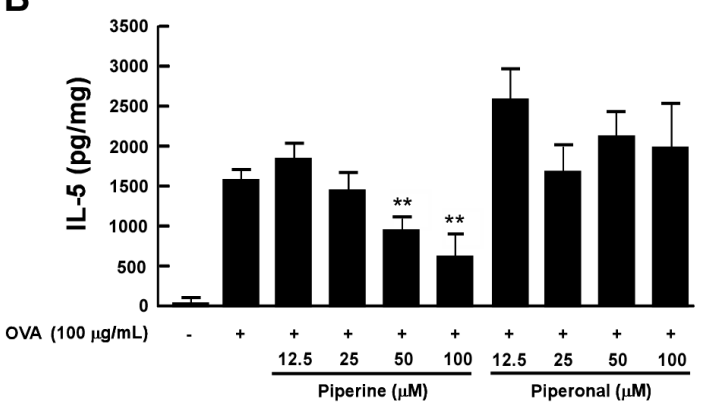

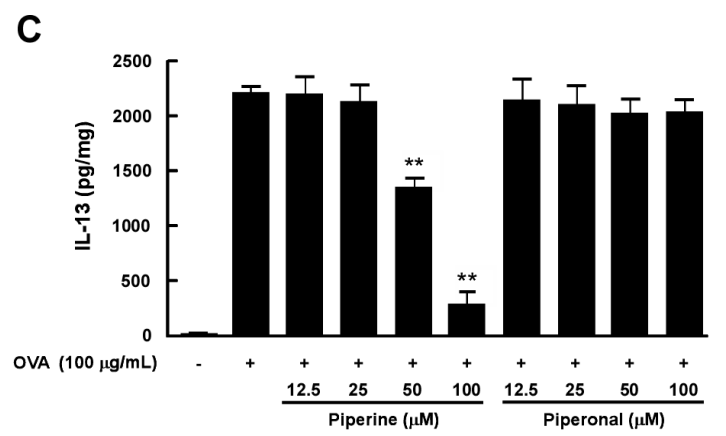

Figure 6. Effect of piperine and piperonal on OVA-induced Th2 cytokine expression ex vivo

Piperine inhibits OVA-induced IL-4 (A), IL-5 (B) and IL-13 (C) production in primary cultured splenocytes. After mice were treated as described for Figure 5A, homogenized single spleen cells spleen were treated with piperine and piperonal in the presence or absence of OVA, and culture media was analyzed by ELISA to detect IL-4, IL-5, and IL-13 cytokine expression. Data are presented as the mean \pm SD of three independent experiments. Asterisks $(*)$ and $(* *)$ indicate significant differences of $p<0.05$ and $p<0.01$, respectively, between groups with and without PFE.

\section{Discussion}

Unregulated immune responses to pathogens cause inflammatory reactions in the host's body. When haptens lower than 500 daltons penetrate the skin, immune defense systems attempt to remove the haptens alone or with the aid of hapten-conjugated proteins (Kaplan et al., 2012). During this step, a variety of immune cells including Langerhans cells, dermal dendritic cells, T lymphocytes, and keratinocytes are involved in the immune response and eventually cause skin inflammation (Honda et al., 2013). Globally, $15-20 \%$ of the general public suffers from the symptoms of ACD including pruritus and pain (Peiser et al., 2012). Although the pharmacological agents cortisol, prednisolone, and dexamethasone are used for the treatment of allergies, inflammation, and autoimmune disorders, the side effects of these drugs have driven attempts to develop more effective immune modulating agents (Berthelot et al., 2013). From a functional food perspective, evidence for the preventive effects of natural compounds against ACD is necessary before such materials can be used in pharmaceutical preparations.

Piper nigrum is widely used as a spice and it has been reported to exhibit various beneficial properties, including antibacterial activity (Paulkumar et al., 2014) and improvements in memory function (Hritcu et al., 2014). Additionally, piperine from Piper nigrum has been observed to exert a suppressive effect on OVA-induced asthma (Kim \& Lee, 2009). However, to date, the fruit of Piper nigrum and its biological effects on ACD remain unclear. Multiple lines of evidence suggest that rodent models are optimal for the development of anti-ACD materials because rodent dermatitis induced by haptens shares common symptoms with human dermatitis (Schneider et al., 2009). Therefore, we adopted a TMA-induced rodent dermatitis model and prednisolone as a positive control to investigate the effects of PFE on TMA-induced dermatitis (Schneider et al., 2009).

We first investigated whether PFE alleviates TMA-induced dermatitis by measuring both ear and epidermal thickness. The results showed that PFE strongly suppressed TMA-induced increases in ear and epidermal thickness, in addition to redness and swelling after oral administration. PFE also strongly suppressed 
TMA-induced $T$ helper cell recruitment into the mouse ear tissue. In our studies, to evaluate the preventive effects of PFE on TMA-induced ACD, we conducted oral administration prior to sensitization by TMA, and the resultant data indicates that PFE exerts a preventive effect toward TMA-induced chronic ACD.

Cytokines from $\mathrm{T}$ helper cells and antibodies from B cells play important roles in allergic responses (Honda et al., 2013). In our screening study, we found that PFE significantly suppresses IgE and IL-4 production (data not shown). We then attempted to determine the effect of PFE on TMA-induced IgE production and cytokine expression in vivo. Although PFE significantly suppressed TMA-induced IgE and mMCP-1 production, its effect was markedly lower than that observed for Th2 cytokine production. We therefore chose to focus on cytokine expression and Th cells as major immune responders in the presence of PFE. Although the dysregulation of Th1 and Th2 cytokines is considered as an important contributor toward the development of ACD, the concept is changing with substantial new findings (Furuzawa-Carballeda et al., 2007). Interestingly, in our studies, IFN gamma, a representative Th1 cytokine, remained unaffected by PFE (data not shown). A previous study that established the chronic TMA rodent model also revealed that prednisolone does not affect TMA-induced IgE production, even though it has a suppressive effect on TMA-induced dermatitis (Schneider et al., 2009). We found that oral administration of PFE strongly suppressed TMA-induced IL-4 and IL-5 production, as well as IL-4 mRNA expression in vivo. A previous study revealed that chronic exposure of TMA to rodent skin elevates Th2 cytokine production, including IL-5, but not Th1 cytokines, such as IFN- $\gamma$ (Hopkins et al., 2005). We also confirmed the effect of PFE on OVA-induced Th2 cytokine expression including IL-4, IL-5, and IL-13. Additionally, Western blot assay results also support our hypothesis that PFE inhibits STAT6 phosphorylation in both primary cultured rodent splenocytes and human keratinocytes. The results indicate that the preventive effect of PFE toward dermatitis is due to the suppression of Th2 cytokine production induced by chronic treatment of TMA.

Piperine was one of the first active compounds isolated from P. nigrum fruit (Scott et al., 2005). It was thus a primary suspect responsible for the effects of PFE in terms of anti-ACD properties. In our study, piperine was observed to exert an inhibitory effect toward OVA-induced Th2 cytokine production, including IL-4, IL-5, and IL-13, but piperonal had no such effect. Although peridine also appears to be an active compound in PFE, further studies are needed to investigate its efficacy and the metabolic pathways involved, because the eventual compound structures present in the human body are dependent upon metabolism and extraction methods.

Taken together, our results suggest that PFE significantly inhibits TMA-induced ACD in mice. This inhibition occurs primarily through the inhibition of Th2 cytokines, occurring via reduced STAT phosphorylation and GATA3 mRNA expression. The therapeutic inhibition of Th2 cytokines by PFE may provide clinical benefits for the more effective treatment of dermatitis. This represents the first report to elucidate aspects of the molecular basis for the anti-ACD effects of PFE.

\section{References}

Berthelot, J. M., Le Goff, B., \& Maugars, Y. (2013). Side effects of corticosteroid injections: what's new? Joint Bone Spine, 80(4), 363-367. http://dx.doi.org/10.1016/j.jbspin.2012.12.001

Bieber, T. (2008). Atopic dermatitis. $N$ Engl $J$ Med, 358(14), 1483-1494. http://dx.doi.org/10.1056/NEJMra074081

Cho, E., \& Cho, S. H. (2013). Effects of Korean red ginseng extract on the prevention of atopic dermatitis and its mechanism on early lesions in a murine model. $J$ Ethnopharmacol, 145(1), 294-302. http://dx.doi.org/10.1016/j.jep.2012.11.006

Choi, J. H., Jin, S. W., Han, E. H., Park, B. H., Kim, H. G., Khanal, T., ... Jeong, H. G. (2014). Platycodon grandiflorum root-derived saponins attenuate atopic dermatitis-like skin lesions via suppression of NF-kappaB and STAT1 and activation of Nrf2/ARE-mediated heme oxygenase-1. Phytomedicine, 21(8-9), 1053-1061. http://dx.doi.org/10.1016/j.phymed.2014.04.011

Eglite, S., Morin, J. M., \& Metzger, H. (2003). Synthesis and secretion of monocyte chemotactic protein-1 stimulated by the high affinity receptor for IgE. $J$ Immunol, 170(5), 2680-2687. http://dx.doi.org/10.4049/jimmunol.170.5.2680

Furuzawa-Carballeda, J., Vargas-Rojas, M. I., \& Cabral, A. R. (2007). Autoimmune inflammation from the Th17 perspective. Autoimmun Rev, 6(3), 169-175. http://dx.doi.org/10.1016/j.autrev.2006.10.002

Gould, H. J., \& Sutton, B. J. (2008). IgE in allergy and asthma today. Nat Rev Immunol, 8(3), 205-217. http://dx.doi.org/10.1038/nri2273 
Hirata, N., Naruto, S., Inaba, K., Itoh, K., Tokunaga, M., Iinuma, M., \& Matsuda, H. (2008). Histamine release inhibitory activity of Piper nigrum leaf. Biol Pharm Bull, 31(10), 1973-1976. http://dx.doi.org/10.1248/bpb.31.1973

Honda, T., Egawa, G., Grabbe, S., \& Kabashima, K. (2013). Update of immune events in the murine contact hypersensitivity model: toward the understanding of allergic contact dermatitis. J Invest Dermatol, 133(2), 303-315. http://dx.doi.org/10.1038/jid.2012.284

Hopkins, J. E., Naisbitt, D. J., Kitteringham, N. R., Dearman, R. J., Kimber, I., \& Park, B. K. (2005). Selective haptenation of cellular or extracellular protein by chemical allergens: association with cytokine polarization. Chem Res Toxicol, 18(2), 375-381. http://dx.doi.org/10.1021/tx049688+

Hritcu, L., Noumedem, J. A., Cioanca, O., Hancianu, M., Kuete, V., \& Mihasan, M. (2014). Methanolic extract of Piper nigrum fruits improves memory impairment by decreasing brain oxidative stress in amyloid beta(1-42) rat model of Alzheimer's disease. Cell Mol Neurobiol, 34(3), 437-449. http://dx.doi.org/10.1007/s10571-014-0028-y

Jin, J. H., Ngoc, T. M., Bae, K., Kim, Y. S., \& Kim, H. P. (2011). Inhibition of experimental atopic dermatitis by rhubarb (rhizomes of Rheum tanguticum) and 5-lipoxygenase inhibition of its major constituent, emodin. Phytother Res, 25(5), 755-759.

Kaplan, D. H., Igyarto, B. Z., \& Gaspari, A. A. (2012). Early immune events in the induction of allergic contact dermatitis. Nat Rev Immunol, 12(2), 114-124.

Kim, B. H., Na, K. M., Oh, I., Song, I. H., Lee, Y. S., Shin, J., \& Kim, T. Y. (2013). Kurarinone regulates immune responses through regulation of the JAK/STAT and TCR-mediated signaling pathways. Biochem Pharmacol, 85(8), 1134-1144. http://dx.doi.org/10.1016/j.bcp.2013.01.005

Kim, S. H., \& Lee, Y. C. (2009). Piperine inhibits eosinophil infiltration and airway hyperresponsiveness by suppressing $\mathrm{T}$ cell activity and Th2 cytokine production in the ovalbumin-induced asthma model. J Pharm Pharmacol, 61(3), 353-359. http://dx.doi.org/10.1211/jpp.61.03.0010

Martin, S. F. (2004). T lymphocyte-mediated immune responses to chemical haptens and metal ions: implications for allergic and autoimmune disease. Int Arch Allergy Immunol, 134(3), 186-198. http://dx.doi.org/10.1159/000078765

Mosmann, T. R., Cherwinski, H., Bond, M. W., Giedlin, M. A., \& Coffman, R. L. (2005). Two types of murine helper T cell clone. I. Definition according to profiles of lymphokine activities and secreted proteins. 1986. J Immunol, 175(1), 5-14.

Murphy, K. M., \& Stockinger, B. (2010). Effector T cell plasticity: flexibility in the face of changing circumstances. Nat Immunol, 11(8), 674-680. http://dx.doi.org/10.1038/ni.1899

Paulkumar, K., Gnanajobitha, G., Vanaja, M., Rajeshkumar, S., Malarkodi, C., Pandian, K., \& Annadurai, G. (2014). Piper nigrum leaf and stem assisted green synthesis of silver nanoparticles and evaluation of its antibacterial activity against agricultural plant pathogens. ScientificWorldJournal, 2014, 829894.

Peiser, M., Tralau, T., Heidler, J., Api, A. M., Arts, J. H., Basketter, D. A., ... Luch, A. (2012). Allergic contact dermatitis: epidemiology, molecular mechanisms, in vitro methods and regulatory aspects. Current knowledge assembled at an international workshop at BfR, Germany. Cell Mol Life Sci, 69(5), 763-781. http://dx.doi.org/10.1007/s00018-011-0846-8

Savinko, T., Karisola, P., Lehtimaki, S., Lappetelainen, A. M., Haapakoski, R., Wolff, H., ... Alenius, H. (2013). ST2 regulates allergic airway inflammation and T-cell polarization in epicutaneously sensitized mice. $J$ Invest Dermatol, 133(11), 2522-2529. http://dx.doi.org/10.1038/jid.2013.195

Schneider, C., Docke, W. D., Zollner, T. M., \& Rose, L. (2009). Chronic mouse model of TMA-induced contact hypersensitivity. J Invest Dermatol, 129(4), 899-907. http://dx.doi.org/10.1038/jid.2008.307

Scott, I. M., Puniani, E., Jensen, H., Livesey, J. F., Poveda, L., Sanchez-Vindas, P., ... Arnason, J. T. (2005). Analysis of Piperaceae germplasm by HPLC and LCMS: a method for isolating and identifying unsaturated amides from Piper spp extracts. J Agric Food Chem, 53(6), 1907-1913. http://dx.doi.org/10.1021/jf048305a

Szabo, S. J., Kim, S. T., Costa, G. L., Zhang, X., Fathman, C. G., \& Glimcher, L. H. (2000). A novel transcription factor, T-bet, directs Th1 lineage commitment. Cell, 100(6), 655-669. http://dx.doi.org/10.1016/S0092-8674(00)80702-3

Tokura, Y. (2010). Extrinsic and intrinsic types of atopic dermatitis. J Dermatol Sci, 58(1), 1-7. 
http://dx.doi.org/10.1016/j.jdermsci.2010.02.008

Zhu, J., Yamane, H., \& Paul, W. E. (2010). Differentiation of effector CD4 T cell populations (*). Annu Rev Immunol, 28, 445-489. http://dx.doi.org/10.1146/annurev-immunol-030409-101212

\section{Copyrights}

Copyright for this article is retained by the author(s), with first publication rights granted to the journal.

This is an open-access article distributed under the terms and conditions of the Creative Commons Attribution license (http://creativecommons.org/licenses/by/3.0/). 